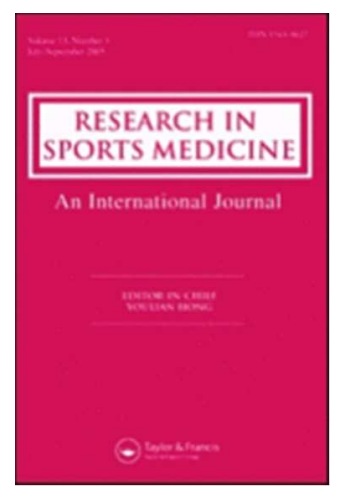

\title{
Kinesiology tape mediates soccer simulated and local peroneal fatigue in soccer players
}

\begin{tabular}{|r|l|}
\hline Journal: & Research in Sports Medicine \\
\hline Manuscript ID & GSPM-2016-0113.R3 \\
\hline Manuscript Type: & Original Research \\
\hline Keywords: & taping techniques, fatigue, balance, soccer \\
\hline
\end{tabular}

SCHOLARONE ${ }^{\mathrm{m}}$

Manuscripts 


\section{Abstract}

4 To investigate the efficacy of kinesiology taping in mediating the influence of fatigue on

5 ankle sprain risk, twelve male soccer players completed single leg dynamic balance trials

6 pre- and post-exercise (soccer-specific protocol, isokinetic ankle inversion/eversion

7 protocol) in each of three counter-balanced taping conditions (no tape, zinc oxide tape ZO,

8 kinesiology tape KT). Balance was quantified as the overall stability index (OSI) and

9 directional stability indices of platform deflection. Soccer-specific fatigue only increased OSI

10 in the no tape condition $(P=0.03)$, with $\mathrm{ZO}$ and $\mathrm{KT}$ trials negating a fatigue affect. Localised

11 fatigue increased OSI in the no tape $(P=0.01)$ and ZO $(P=0.05)$ trials, with no increase in

12 the KT trial. A similar pattern was observed in medio-lateral and anterio-posterior balance

13 indices. Kinesiology tape mediates soccer simulated and local peroneal fatigue, with

14 practical implications for epidemiological observations of increased injury risk during the

15 latter stages of match-play.

\section{INTRODUCTION}

18 Ankle sprain injury is common in many sports, and exacerbated in those sports with a multi-

19 directional and reactive activity profile. In an audit of soccer injuries, $54 \%$ of ankle sprain 20 injuries were observed during tackle scenarios (Woods, Hawkins, Hulse \& Hodson, 2003),

21 but the $39 \%$ sustained during non-contact mechanisms offer the best scope for prevention.

22 Hawkins et al. (1999) identified that an average of 14.6 days per season are lost due to

23 injury in soccer, with a high re-injury rate and greater severity on reoccurrence. Twice as

24 many ankle sprains were sustained in matches than training, and a disproportionately large 
1 proportion of match sprains were incurred during the latter stages of match-play (Woods et

2 al., 2003), highlighting fatigue as a risk factor.

3 Fatigue is a complex phenomenon and has been categorised into central systemic (altered

4 central nervous system transmission or recruitment) or local peripheral (impairment of

5 functional transmission, muscle electrical activity and activation) fatigue (Kirkendall, Junge,

6 \& Dvorak, 2010). To investigate the influence of fatigue on the aetiology of injury, the

7 exercise models must be valid as injury is commonly associated with mechanical load failure

8 of tissue (Bahr \& Holme, 2005). Soccer-specific exercise protocols based on the velocity

9 profile of match-play can simulate the intermittent and irregular activity profile of soccer

10 and have previously been used to quantify changes in functionally challenging movements

11 such as balance and agility (Greig, 2009; Greig \& Walker-Johnson, 2007; Lohkamp et al.,

12 2009). Local, peripheral fatigue has typically been modelled using isokinetic dynamometry

13 trials (Lin, Lin, Tsai, \& Ling, 2008; South \& George, 2007), targeting the contribution of

14 peroneal latency to inversion control (Gribble, Hertal, Denegar, \& Buckley, 2004).

15 The influence of fatigue on injury risk has been attributed to diminishing neuromuscular

16 control and proprioception accuracy (Gurney, Milani, \& Pederson, 2000), providing a focus

17 for preventive strategies. The importance of preventative measures to potentially decrease

18 ankle injury incidence and recurrence in sport have been well documented (Fong, Hong,

19 Chan, Yung, \& Chan, 2007). Ankle injury prevention has been given considerable attention

20 with strategies often incorporating traditional taping methods (e.g. Raymond, Nicholson,

21 Hiller, \& Refshauge, 2012; Stryker, Di Trani, Swanik, Glutting \& Kaminiski, 2016) to decrease

22 biomechanical joint range of movement (ROM) or to stimulate kinaesthetically through

23 cutaneous mechanoreceptors (Verhagen \& Bay, 2010). More recently the potential of

24 kinesiology tape has been explored in promoting proprioception and neuromuscular 
1 activation, both as a preventative measure (Briem, Eythorsdottir, Magnusdottir, Palmarsson,

2 Runarsdottir, \& Sveinsson, 2011) and as a treatment modality (Simon, Garcia \& Docherty,

3 2014; Szymura, Maciejczk, Wiecek, Maciejczyk, Wiecha, \& Ochalek, 2016). It is commonly

4 proposed that cutaneous stimulation promotes joint stability and muscle activation,

5 however the type of tape and the application influence the efficacy (Thedon, Mandrick,

6 Foissac, Mottet \& Perrey, 2011; Csapo \& Alegre, 2015; Trecroci, Formenti, Rossi, Esposito \&

7 Alberti, 2015). The aim of the current study was to determine if taping offers the potential

8 to mediate the negative effects of fatigue on balance performance, with practical

9 implications for players and medical practitioners based on ankle sprain epidemiology.

11 MATERIALS AND METHOD

12 Participants

1312 male semi-professional soccer players (age $21.6 \pm 0.7$ years; height $181.6 \pm 9.3 \mathrm{~cm}$; body

14 mass $76.7 \pm 5.2 \mathrm{~kg}$; fat mass $10.8 \pm 1.7 \mathrm{~kg}$ ), participated in the present study. All players 15 were contracted to play for clubs competing in tier 5 or 6 of the English Football Association.

16 All testing was conducted following a 6 week pre-season period, and in the 3 weeks prior to

17 the commencement of the competitive season. Inclusion criteria required that all players

18 were currently completing a minimum of two club training sessions and one match per

19 week to ensure standardisation of physical status. Additional criteria required that players

20 were injury free in the previous 3 months, and specifically free from ankle and knee injury in

21 the previous 6 months. Players who exhibited neurologic or balance disorders, or chronic

22 ankle instability as determined by the Cumberland Ankle Instability Tool were excluded from

23 the study. Players with skin allergies were also excluded from participation. This excluded

24 an initial 8 participants recruited. Participants were fully informed of the demands and 
1 possible risks associated with the investigation and were given the opportunity to withdraw

2 from the study at any time. All tests were carried out at between 14:00 to 16:00 $\mathrm{h}$ to negate

3 circadian influences, and in accord with regular competition time. Each participant provided

4 written informed consent prior to the study. The study conformed to the standards set by

5 the Declaration of Helsinki and was approved by the Institutional ethics committee (Harris \&

6 Atkinson, 2013).

\section{Experimental Design}

8 Participants completed a single legged balance task on the Biodex Stability System (BSS) at

9 Level 2. The BSS has 8 levels, with Level 8 providing the most stable surface, and thus Level

102 was chosen as a functionally challenging level of stability for these participants. Testing

11 was conducted on the dominant leg, defined as the preferred kicking leg (Hawkins et al.,

12 1999) as epidemiological data suggests a higher incidence of injury to this side (Woods et al.,

13 2003). Balance trials were completed pre- and post-exercise, with two exercise protocols

14 being used. Three taping conditions were used in the current study, such that each player

15 completed a total of six experimental conditions. The taping (3) and exercise protocol (2)

16 conditions were order effect counter-balanced and randomised in delivery, and separated

17 by a minimum of $72 \mathrm{hrs}$, with testing completed over a 3 week period. Single leg balance

18 performance on the BSS task was quantified as overall stability index (OSI), and further sub-

19 divided into the directional components of medial/lateral (ML) and Anterior/Posterior (AP)

20 balance. Prior to experimental trials, participants attended the laboratory to complete

21 familiarisation sessions on both exercise protocols and the BSS task. Baseline, pre-exercise

22 scores for BSS task performance were also attained in each tape condition.

23 The three taping conditions used in the present study are categorised as a no-tape, control

24 condition (NT), zinc oxide tape (ZO), or kinesiology tape (KT). In the ZO condition, a 
1 traditional taping anchor and 3 stirrups method was used, designed to provide lateral

2 ligament support (Figure 1a). In the KT condition (RockTape, Essex, UK), designed for

3 muscle facilitation application of the peroneals, I strip, after the initial resting position, 50\%

4 stretch was applied with the patient in dorsiflexion and eversion when taping post anchor

5 application proximal to distal (Figure 1b), in accordance with KT® guidelines (Kase, Wallis, \&

6 Kase, 2003). With both taping techniques the area was shaved and prepared prior to

7 application and the procedure for tape activation and removal was also adhered to (Kase et

8 al., 2003). The same appropriate footwear was worn for each session, reducing the surface

9 interaction effect.

10

11

12

13 The exercise protocols used in the present study were categorised as a 'soccer simulated,'

14 soccer-specific treadmill protocol (Greig et al., 2006) and a 'local' isokinetic ankle inversion-

15 eversion protocol (South \& George, 2007). The 'soccer simulated' fatigue protocol is based

17 conjunction with the BSS task (Greig \& Walker-Johnson, 2007). A 45min period of irregular

18 and intermittent running was completed, replicating the $1^{\text {st }}$ half of a soccer match, on the

19 basis that tape would be re-applied during the half-time period. The 'local' fatigue protocol

20 comprised 3 sets of 30 repetitions of isokinetic ankle inversion/eversion at $60^{\circ} \cdot \mathrm{s}^{-1}$, with a 10

21 sec rest applied between sets. This protocol design has previously been used to induce

22 fatigue within the peroneal muscle group, defined as when performance reached $50 \%$ of the

23 subjects pre exercise peak torque (South \& George, 2007).

\section{Statistical Analysis}


1 A two-factor (tape $\mathrm{x}$ exercise protocol) general linear model with repeated measures was

2 used to compare between trials, supplemented with values of effect size (ES) to provide a

3 measure of meaninglfulness. The assumptions associated with a repeated measures general

4 linear model (GLM) were assessed to ensure model adequacy. To assess residual normality

5 for each dependant variable, q-q plots were generated using stacked standardised residuals.

6 Scatterplots of the stacked unstandardized and standardised residuals were also utilised to

7 assess the error of variance associated with the residuals. Mauchly's test of sphericity was

8 also completed for all dependent variables, with a Greenhouse Geisser correction applied if

9 the test was significant. The aforementioned measures did not violate any of the

10 assumptions, therefore inferential analyses were performed. Inferential analyses were

11 performed using a repeated measure general linear model (GLM) to examine differences in

12 the physical response between the speed, limb and contraction over time. Where significant

13 main effects were observed, post hoc pairwise comparisons with a Bonferonni correction

14 factor were applied. All statistical analysis was completed using PASW Statistics Editor 22.0

15 for windows (SPSS Inc, Chicago, USA). Statistical significance was set at $P \leq 0.05$. Statistical

16 significance was set at $P \leq 0.05$, and all data are presented as mean \pm standard deviation.

18 RESULTS

19 Figure 2 summarises the influence of tape condition and exercise protocol on the overall

20 stability index (OSI). Pre-exercise, and relative to the control condition $(1.75 \pm 0.35)$, both

21 the ZO $(1.39 \pm 0.31, P=0.13, \mathrm{ES}=1.06)$ and $\mathrm{KT}(1.30 \pm 0.34, P=0.08, \mathrm{ES}=1.32)$

22 interventions had a positive, but non-significant effect on OSI relative to the control

23 condition. There was no statistically significant distinction between the taping

24 interventions. The soccer-specific fatigue protocol had a significant detriment on OSI only in 
1 the control condition $(2.14 \pm 0.59, P=0.03, E S=0.98)$ relative to pre-exercise measures.

2 The localised fatigue protocol produced a significant increase in OSI in both the control (2.93

$3 \pm 0.76, P=0.01, \mathrm{ES}=1.55)$ and $\mathrm{ZO}(2.14 \pm 0.69, P=0.05, \mathrm{ES}=1.09)$ trials relative to baseline.

7 A similar temporal pattern was observed in both the anterio-posterior (Figure 3) and medio-

8 lateral (Figure 4) directional indices of balance. In the anterio-posterior plane, the soccer-

9 specific protocol only produced a significant increase in API in the control condition $(P=$

$100.05, E S=0.80$ ), whilst the localised fatigue protocol significantly increased API in the

11 control $(P=0.04, \mathrm{ES}=0.87)$ and $\mathrm{ZO}$ trials $(P=0.05, \mathrm{ES}=0.72)$. MLI was not significantly

12 influenced by the soccer-specific trial, but the localised protocol induced a significant

13 increase in the control $(P=0.02, \mathrm{ES}=1.42)$ and $\mathrm{ZO}(P=0.05, \mathrm{ES}=1.31)$ trials.

14

\section{DISCUSSION}

** Insert Figure $3 \& 4$ near here **

Kinesiology tape demonstrated the potential to mediate a fatigue-effect observed in balance performance following both localised and soccer-specific exercise protocols. The KT application was also significantly better than the ZO application following localised fatigue, indicative that the ergogenic effect is greatest where needed the most. During the localised fatigue protocol an increased load is applied directly through the ankle complex, in comparison to the load distribution within the lower body during the soccer-specific running protocol. These findings have implications for injury prevention given the epidemiological 
1 observations of increased ankle sprain incidence during the latter stages of soccer match-

2 play (Woods et al., 2003). However, the mediation of fatigue in the current study is contrary

3 to that observed in some contemporary research, although direct comparisons should be

4 treated with caution due to methodological differences, not least the target joint and taping

5 application. For example, Zanca et al. (2015) reported that kinesiology tape application did

6 not improve shoulder JPS acuity following repeated elevation in the scapular plane. Aarseth

7 et al. (2015) also showed reduced JPS acuity at $90^{\circ}$ of elevation, but showed no impairment

8 at $50^{\circ}$ or $110^{\circ}$, suggesting an angle-specific influence. In lumbar extensor muscular

9 endurance tests, Alvarez-Alvarez et al. (2014) did observe improved time to failure with

10 kinesiology tape application in healthy subjects, whilst Hagen et al. (2015) observed an

11 improvement less than measurement error in patients with low back pain. The literature to

12 date is thus equivocal, and efficacy is likely to be a function of a myriad of experimental

13 design features including joint, exercise intervention, taping application, and outcome

14 measure.

15 The mechanistic underpinning for the mediating benefits of KT may be due, in part, to the

16 adhesive properties of the tape which create a positive thermal and tactile effect. Thedon

17 et al. (2011) suggested that impaired postural performance might be compensated by

18 increased skin stimulation via kinaesthetic stimulation of the cutaneous mechanoreceptors.

19 Simoneau et al. (1997) associated this cutaneous receptors stimulation with enhanced

20 motor activity, joint stability and muscle facilitation. The peroneal musculature has a

21 primary role in ankle eversion, assisting plantar flexion, and thus the KT peroneal muscle

22 facilitation application creates a multi-planar cutaneous benefit. This has been postulated

23 to be attributable to altered speed and duration of peroneal activation (Wilkerson, 2002).

24 Due to the anatomical position of the peroneals, the tape passes on to the sole of the foot, 
1 containing the subcutaneous receptors, and influencing the neuromuscular control of the

2 whole kinetic chain due to the contribution to the control of stance and locomotion (Inglis

3 et al., 2002). KT might assist in stimulation of these receptors to assist the kinetic chain

4 generally, and ankle kinematics, as has previously been observed at the shoulder (Hsu et al.,

5 2009).

6 Traditional taping has commonly been used as a preventative intervention, but in the

7 present study ZO did not mediate local fatigue, with significant impairment in balance

8 performance post-exercise. This finding is consistent with the majority of studies,

9 suggesting a $50-90 \%$ decrease in support after application in a variety of sports due to

10 moisture accumulation weakening the tape and the mobility of skin decreasing the taping

11 efficacy over time (Delahunt, O’Driscoll, \& Moran 2009; Tregouet, Merland, Horodyski,

12 2013). In contrast, ZO did mediate API and MLI after the soccer-specific protocol might be

13 indicative that tape application assists joint restriction creating a reduction in inversion

14 amplitudes and angular velocities whilst allowing joint protection through reflex responses

15 under load (Delahunt et al., 2009; Hubbard \& Cordova, 2010).

16 These results indicate that kinesiology taping in healthy participants can mediate fatigue,

17 potentially as a preventative method to reduce the rate of peroneal latency and subsequent

18 ankle inversion injury risk. However, it must be considered that the findings of the present

19 study should not be generalised beyond the specific population, fatigue protocols, and

20 taping interventions used. The participants were selected so as to be appropriate to the

21 chosen soccer-specific fatigue protocol. This protocol has previously been shown to induce

22 changes in dynamic balance (Greig \& Walker-Johnson, 2007), but alternate free running

23 protocols might be considered to pose a more functionally valid simulation given the multi-

24 directional nature of soccer locomotion. Soccer-specific activities such as kicking, turning, 
1 and tackling are negated in such experimental protocols in order to attain greater

2 experimental control, but their influence is worthy of consideration given their association

3 with ankle sprain injury mechanism. Extending the exercise protocol to $90 \mathrm{mins}$ would also

4 enable an investigation into the efficacy of kinesiology tape for the duration of a soccer

5 match, and help inform strategy in terms of optimum application. Acknowledging

6 limitations including confounding and extrinsic factors, future studies should consider the

7 tape application influence on chronic and functional ankle instability, movement dynamics

8 and altered loading. Study designs might also be developed to contrast the application of

9 KT with other prevention programs and proprioception exercises. Limb dominance might be

10 an additional variable worthy of analysis, given epidemiological observations of bi-lateral

11 variation in incidence (Woods et al., 2003).

12 In conclusion, both traditional zinc oxide and kinesiology taping applications were observed

13 to negate a fatigue-effect in dynamic stability following 45 mins of soccer-specific activity.

14 Kinesiology tape was also able to significantly reduce the influence of fatigue localised to

15 ankle inversion-eversion. In terms of practical implications, if KT can act as a preventative

16 strategy without joint restriction or biomechanical change the application needs to be

17 considered as an alternative to current and widely used traditional taping methods in ankle

18 inversion prevention. Equivocal findings across an expanding literature base have yet to

19 fully determine the biomechanical and physiological mechanisms of the potential benefits to 20 performance.

22 ACKNOWLEDGEMENTS

23 No financial support was provided for this study. 


\section{REFERENCES}

1. Aarseth, L.M., Suprak, D.N., Chalmers, G.R., Lyon, L., Dahlquist, D.T. (2015) Kinesio Tape and Shoulder-Joint Position Sense. Journal of Athletic Training 50(8), 785-791.

2. Alvarez-Alvarez, S., San Jose F.G-M., Rodriguez-Fernandez, A.L., Gueita-Rodriguez, J., Waller, B.J. (2014) Effects of Kinesio Tape in low back muscle fatigue: Randomized, controlled, double blinded clinical trial on healthy subjects. Journal of Back and Musculoskeletal Rehabilitation 27, 203-212.

3. Bahr, R. \& Holme, I. (2005) Risk factors for sports injuries - a methodological approach. British Journal of Sports Medicine 37(5), 384-92.

4. Briem, K., Eythorsdottir, H., Magnusdottir, R.G., Palmarsson, R., Runarsdottir, T., Sveinsson, T. (2011) Effects of kinesiotape compared with nonelastic sports tape and the untaped ankle during a sudden inversion perturbation in male athletes. Journal of Orthopaedic Sports Physical Therapy 41(5), 328-335.

5. Csapo, R., \& Allegre, L.M., (2015) Effect of kinesiology taping on skeletal muscle strength- a meta-analysis of current evidence. Journal of Sport and Medicine in Sport 18 (4) $450-456$

6. Delahunt, E., O’Driscoll, J., Moran, K. (2009) Effects of taping and exercise on ankle joint movement in subjects with chronic ankle instability: a preliminary investigation. Archives Physical Medicine \& Rehabilitation 90(8), 1418-1422.

7. Fong, D.T.P., Hong, Y., Chan, L.K., Yung, P.S.H., Chan, K.M., (2007) A systematic review on ankle injury and ankle sprain in sports. Sports Medicine 37(1), 73-94

8. Greig, M., (2009) The influence of soccer specific activity on the kinematics of an agility sprint. European Journal of Sport Science 9(1), 23-33 
1 9. Greig, M.P., McNaughton, L.R., Lovell, R.J., (2006) Physiological and mechanical response to soccer specific intermittent activity and steady state activity. Research in Sports Medicine 14, 29-52

10. Greig, M., \& Walker-Johnson, C. (2007) The influence of soccer-specific fatigue on functional stability. Physical Therapy in Sport 8, 185-190

11. Gribble, P., Hertal, J., Denegar, C.R., Buckley, W.E. (2004) The effects of fatigue and

12. Gurney, B., Milani, J., Pederson, M.E. (2000) Role of fatigue on proprioception of the ankle Journal of Exercise Physiology 3(1), 8

13. Hagen, L., Hebert, J.J., Dekanich, J., Koppenhaver, S. (2015) The effect of elastic therapeutic taping on back extensor muscular endurance in patients with low back pain: A randomized, controlled, crossover trial. Journal of Orthopaedic and Sports Physical Therapy 45(3), 214-218.

14. Harris, D.J., \& Atkinson, G. (2013) Ethical standards in sport and exercise science research. International Journal of Sports Medicine 34, 1025-1028

15. Hawkins, R.D., \& Fuller, C.W (1999) A prospective epidemiological study of injuries in four English professional football clubs. Br J Sports Med 33, 196-203

16. Hsu, Y.H., Chen, W.Y., Lin, H.C., Wang, W.T.J., Shih, Y.F. (2009) The effects of taping on scapular kinematics and muscle performance in baseball players with shoulder impingement syndrome. Jl Electromyography \& Kinesiology 19 (6) 1092-1099

17. Hubbard, T.J., \& Cordova, M. (2010) Effect of ankle taping on mechanical laxity in chronic ankle instability. Foot \& Ankle International 31(6), 499-504 
18. Inglis, J.T., Kennedy, P.M., Wells, C., Chila., R (2002) The role of cutaneous receptors in the foot. Adv Exp Med Biol 508, 111-117

19. Kase, K., Wallis, J., \& Kase T. (2003) Clinical therapeutic applications of the

22. Raymond, J., Nicholson, L.L., Hiller, C.E., \& Refshauge, K. (2012) The effect of ankle taping or bracing on proprioception in functional ankle instability; A systematic review and meta analysis. Journal of Science Medicine in Sport 15, 386-392.

23. Simon, J., Garcia, W., \& Docherty, C. (2014) The effect of kinesiology taping on force sense in people with functional ankle instability. Clinical Journal of Sports Medicine 24(4) $289-294$

24. Simoneau, G.G., Degner, R.M., Kramper, C.A., Kittleson, K.H. (1997) Changes in ankle joint proprioception resulting from strips athletic tape applied over the skin. J Ath Train 3 (2) 141-147

25. South, M., \& George, K.P. (2007) The effect of peroneal muscle fatigue on ankle joint position sense. Physical Therapy in Sport 8(2): 82-87.

26. Stryker, S.M., Di Trani, A.M., Swanik, C., Glutting, J.J. \& Kaminski, T.W. (2016) Assessing performance, stability, and cleat comfort/support in collegiate club soccer players using prophylactic ankle taping and bracing. Research in Sports Medicine. 24 (1) $39-53$ 
27. Szymura, J., Maciejczyk., Wiecej, M., Maciejczyk, G., Wiecha, S., Ochalek., K., Kepinska, M \& Szygula, Z. (2016) Effects of kinesio taping on anaerobic power recovery after eccentric exercise. Research in Sports Medicine. 24 (3) 242-253

28. Thedon, T., Mandrick, K., Foissac, M., Mottet, D., Perrey, S. (2011) Degraded postural performance after muscle fatigue can be compensated by skin stimulation. Gait Posture 33, 686-689.

29. Trecroci, A., Formenti, D., Rossi, A., Esposito, F., \& Alberti, G. (2015) Acute effects of kinesio taping on a $6 \mathrm{~s}$ maximal cycling sprint performance. Research in Sports Medicine, 25:1 48-57

30. Tregouet, P., Merland, F., Horodyski, M.B. (2013) A comparison of the effects of ankle taping styles on biomechanics during ankle inversion. Annals of Physical and Rehabilitation Medicine 56, 113-122.

31. Verhagen, E.L.M., \& Bay, K. (2010) Optimising ankle sprain prevention: a critical review and practical appraisal of the literature. British Journal of Sports Medicine 44(15), $1082-8$.

32. Wilkerson, G., (2002) Biomechanical and neuromuscular effects of ankle taping and bracing. Journal of Athletic Training 37 (4) 436-445

33. Woods, C., Hawkins, R.D., Hulse, M., Hodson, A. (2003) The Football Association Medical Research Programme: an audit of injuries in professional football - an analysis of ankle sprains. British Journal of Sports Medicine 37, 233-238.

34. Zanca, G.G., Mattiello, S.M., Karduna, A.R. (2015) Kinesio taping of the deltoid does not reduce fatigue induced deficits in shoulder joint position sense. Clinical Biomechanics 30(9), 903-907.

URL: http://mc.manuscriptcentral.com/gspm E-Mail: hongyoulian@gmail.com 
1

2

3

4

5

6

7

8

9

10

11

12

13

14

15

16

17

18

19

20

21

22

23

24

25

26

27

28

29

30

31

32

33

34

35

36

37

38

39

40

41

42

43

44

45

46

47

48

49

50

51

52

53

54

55

56

57

58

59

60

\section{LEGEND TO FIGURES}

2 Figure 1. Application of the $\mathrm{ZO}$ and $\mathrm{KT}$ interventions.

3 Figure 2. The influence of tape condition on the fatigue response in Overall Stability.

4 Figure 3. The influence of tape and trial type on the Anterio-Posterior balance index.

5 Figure 4. The influence of tape and trial type on the Medio-Lateral balance index. 6 

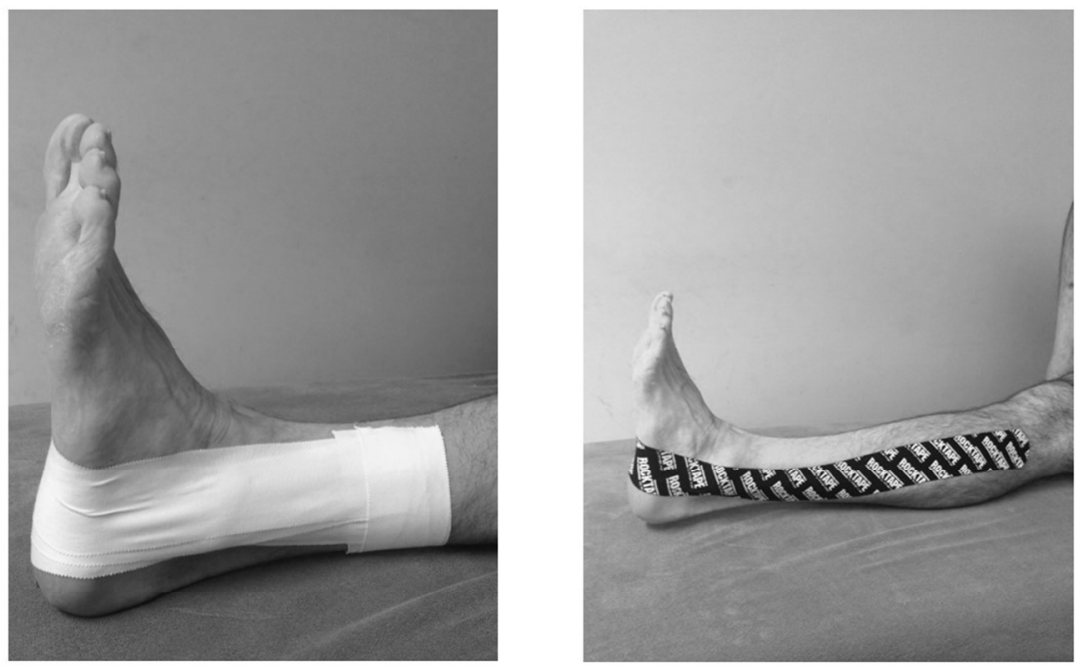

Figure 1. Application of the $\mathrm{ZO}$ and $\mathrm{KT}$ interventions.

$338 \times 190 \mathrm{~mm}(96 \times 96 \mathrm{DPI})$ 
- Pre שPost-Central \& Post-Local

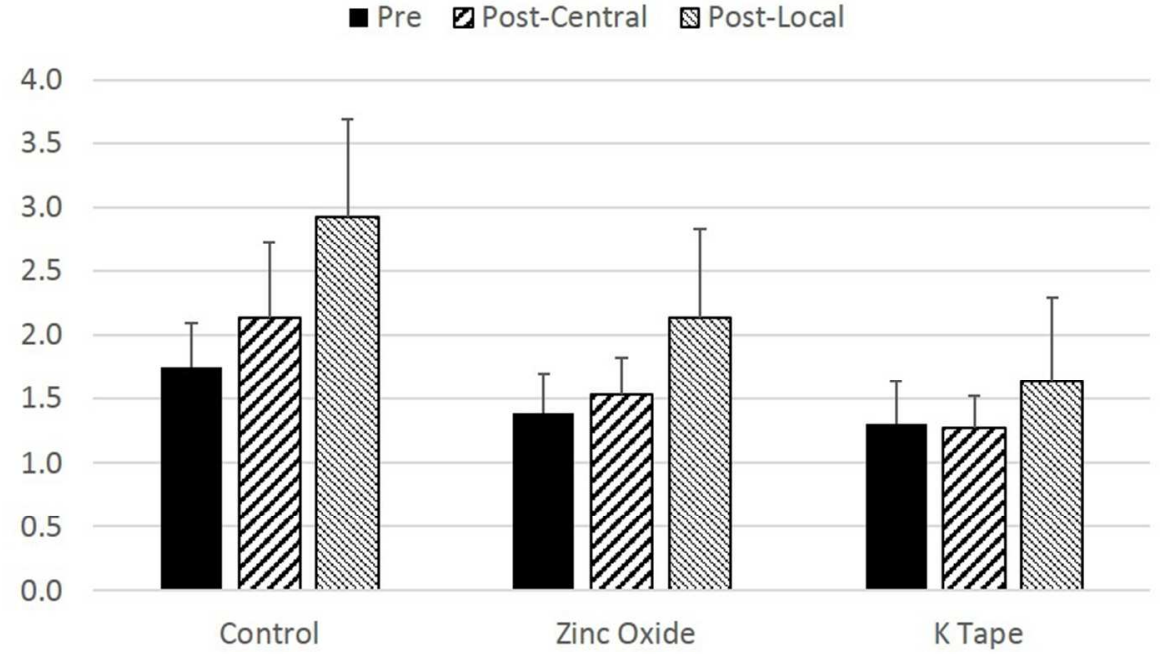

Figure 1. The influence of tape condition on the fatigue response in Overall Stability.

Figure 2. The influence of tape condition on the fatigue response in Overall Stability. $166 \times 103 \mathrm{~mm}(150 \times 150 \mathrm{DPI})$ 


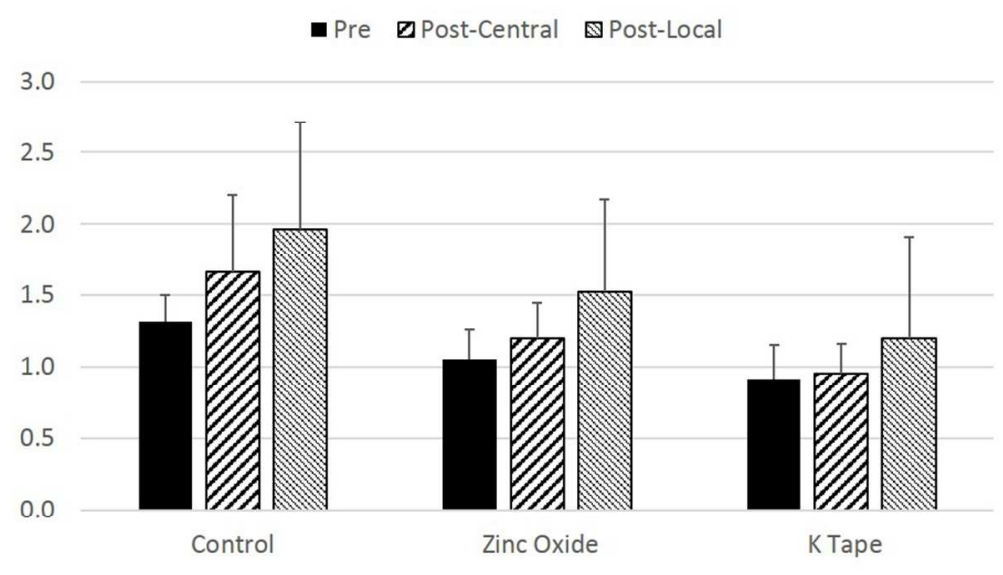

Figure 2. The influence of tape and trial type on the Anterio-Posterior balance index.

Figure 3. The influence of tape and trial type on the Anterio-Posterior balance index. $199 \times 100 \mathrm{~mm}(150 \times 150 \mathrm{DPI})$ 


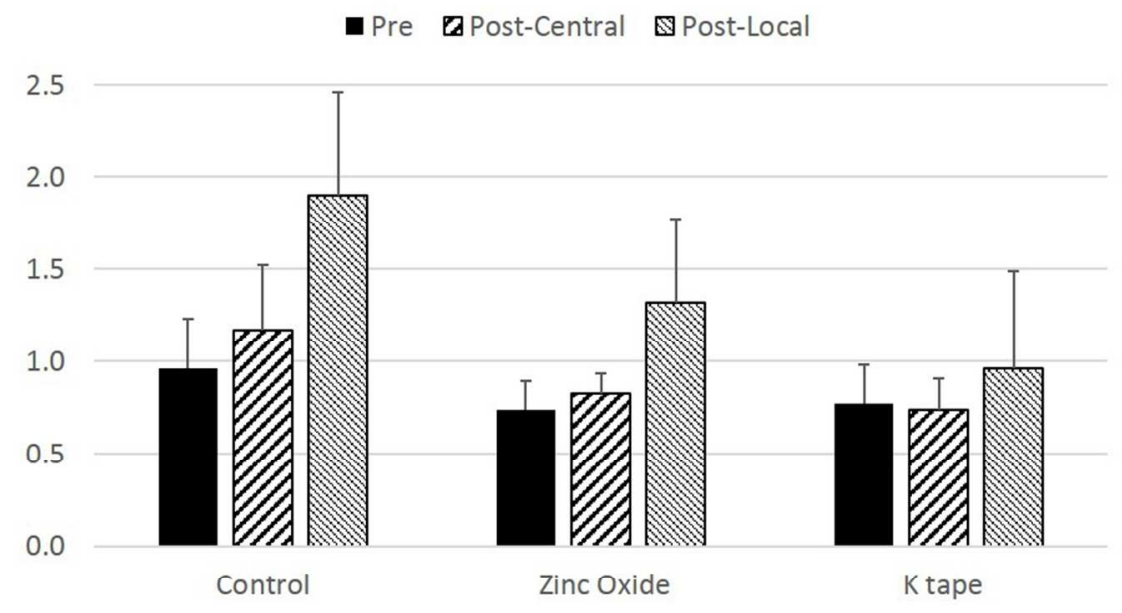

Figure 3. The influence of tape and trial type on the Medio-Lateral balance index.

Figure 4. The influence of tape and trial type on the Medio-Lateral balance index.

$$
176 \times 96 \mathrm{~mm}(150 \times 150 \mathrm{DPI})
$$


Response to Review 1:

Many thanks for the comments and feedback to the revised manuscript submission. We greatly appreciate the opportunity to improve the quality of our submission, and its usefulness to the readers. In particular the suggestion of additional citations we found particularly helpful.

There follows a summary of revisions made to the manuscript in response to Reviewer 1 , and changes on the revised manuscript are highlighted in red font for clarity and ease of review. Of note the inclusion of new references has changed the numerical ordering, and this has been amended throughout the manuscript.

Suggested references have been reviewed and integrated within the revised manuscript (main body of text). We have also updated the reference list as stated above.

Acute effects of kinesio taping on a $6 \mathrm{~s}$ maximal cycling sprint performance, Athos Trecroci, et al., 25(1):48-57, 2017 | DOI: 10.1080/15438627.2016.1258644

In Text p3 L6

Reference 29

Effects of kinesio taping on anaerobic power recovery after eccentric exercise, Jadwiga Szymura, et al., 24(3):257-268, 2015 | DOI: 10.1080/15438627.2016.1202827

In Text p3 L3

Reference 26

Assessing performance, stability, and cleat comfort/support in collegiate club soccer players using prophylactic ankle taping and bracing, Sean M. Stryker, et al. , 24(1):39-53, 2016 |

DOI: 10.1080/15438627.2015.1126274

In Text p2 L21

Reference 27

In addition, and upon reflection of previous comments, we have also slightly amended the manuscript in relation to the tape application, we hope to further increase clarity for the reader.

Amended p5 L3 